\title{
On the Convergence of Exponential Operators- the Zassenhaus Formula, BCH Formula and Systematic Approximants
}

\author{
Masuo Suzuki ${ }^{\star}$ \\ Theoretical Physics Institute, The University of Alberta, Edmonton, Canada T6G 2J1
}

\begin{abstract}
The convergence of the Zassenhaus formula is proven under an appropriate condition as well as for other exponential operators such as the Baker-Campbell-Hausdorff formula.
\end{abstract}

\section{Introduction}

In a previous paper [1], new systematic approximants have been proposed for exponential functions, operators and inner derivation $\delta_{H}$. Remainders of systematic approximants have been evaluated explicitly. In particular, the following $n-m$ approximant $f_{n, m}(A, B)$ of the exponential operator $\exp (A+B)$ is useful in quantum physics [1]:

$$
f_{n, m}(A, B)=\left(e^{A / n} e^{B / n} e^{C_{2} / n^{2}} \ldots e^{n^{-m} C_{m}}\right)^{n} .
$$

Here the coefficients $\left\{C_{n}\right\}$ are polynomials in the operators $A$ and $B$, which appear in the Zassenhaus formula [1-3]. It has been proven in [1] that $\lim _{n \rightarrow \infty} f_{n, m}(A, B)$ $=\exp (A+B)$ for any set of operators $A$ and $B$ in a Banach algebra. The case $m=1$ yields Trotter's formula $[4] \exp (A+B)=\lim [\exp (A / n) \exp (B / n)]^{n}$, which has been the keystone of Monte Carlo simulations of quantum spin systems [5-7].

The main purpose of the present paper is to prove that

$$
\lim _{m \rightarrow \infty} f_{n, m}(A, B)=\exp (A+B)
$$

for any fixed value of $n$, under an appropriate condition. The case $n=1$ yields the Zassenhaus formula. That is, the proof of (1.2) is essentially reduced to that of the convergence of the Zassenhaus formula. The latter is given in Section 2, and Equation (1.2) is proven in Section 3. The convergence of the Baker-CampbellHausdorff formula and related exponential operators is investigated in

* On leave of absence from Department of Physics, Faculty of Science, The University of Tokyo, Hongo, Bunkyo-ku, Tokyo, Japan 
Section 4. These formulae have been used frequently in studying critical phenomena of quantum systems on the basis of the renormalization group approach [8-11].

\section{The Zassenhaus Formula}

The Zassenhaus formula is the following formal expansion (i.e., an infinite product of exponential operators):

$$
e^{\lambda(A+B)}=e^{\lambda A} e^{\lambda B} e^{\lambda^{2} C_{2}} e^{\lambda^{3} C_{3}} \ldots,
$$

where $\left\{C_{n}\right\}$ are defined recursively ${ }^{1}$ as

$$
\begin{aligned}
& C_{2}=\frac{1}{2}\left[\frac{\partial^{2}}{\partial \lambda^{2}}\left(e^{-\lambda B} e^{-\lambda A} e^{\lambda(A+B)}\right)\right]_{\lambda=0}=\frac{1}{2}[B, A], \\
& C_{3}=\frac{1}{3 !}\left[\frac{\partial^{3}}{\partial \lambda^{3}}\left(e^{-\lambda^{2} C_{2}} e^{-\lambda B} e^{-\lambda A} e^{\lambda(A+B)}\right)\right]_{\lambda=0}=\frac{1}{3}\left[C_{2}, A+2 B\right],
\end{aligned}
$$

and in general

$$
C_{n}=\frac{1}{n !}\left[\frac{\partial^{n}}{\partial \lambda^{n}}\left(e^{-\lambda^{n-1} C_{n-1}} \ldots e^{-\lambda^{2} C_{2}} e^{-\lambda B} e^{-\lambda A} e^{\lambda(A+B)}\right)\right]_{\lambda=0} .
$$

We have the following Theorem.

Theorem 1. For any set of operators $A$ and $B$ in a Banach algebra,

$$
\left\|e^{\lambda(A+B)}-e^{\lambda A} e^{\lambda B} \ldots e^{\lambda^{n} C_{n}}\right\| \leqq \delta_{n}[|\lambda|(\|A\|+\|B\|)] \exp [I(|\lambda|)],
$$

where $\delta_{n}(x)$ and $I(x)$ are defined in (2.15) and (2.7), respectively, and $\lim _{n \rightarrow \infty} \delta_{n}(x)=0$ for $0 \leqq x \leqq \ln 2-\frac{1}{2}$. For $|\lambda|(\|A\|+\|B\|) \leqq \ln 2-\frac{1}{2}$,

$$
\lim _{n \rightarrow \infty} e^{\lambda A} e^{\lambda B} e^{\lambda^{2} C_{2}} \ldots e^{\lambda^{n} C_{n}}=e^{\lambda(A+B)} .
$$

Proof. First we prove the convergence of the series defined by

$$
I(\lambda) \equiv \sum_{n=1}^{\infty} \lambda^{n}\left\|C_{n}\right\| ; \quad\left\|C_{1}\right\| \equiv\|A\|+\|B\|
$$

under the above condition. For this, note that

$$
\left\|C_{n}\right\| \leqq \frac{1}{n !}\left(\frac{\partial^{n}}{\partial \lambda^{n}} \exp \left[2 \lambda(\|A\|+\|B\|)+\lambda^{2}\left\|C_{2}\right\|+\ldots+\lambda^{n-1}\left\|C_{n-1}\right\|\right]\right)_{\lambda=0} .
$$

In particular, we have

$$
\begin{aligned}
\left\|C_{2}\right\| & \leqq\left(\frac{1}{2 !} \frac{\partial^{2}}{\partial \lambda^{2}} \exp [2 \lambda(\|A\|+\|B\|)]\right)_{\lambda=0}=a_{2}(\|A\|+\|B\|)^{2} \\
a_{2} & \equiv 2
\end{aligned}
$$

1. Equation (3.17) in [1] should read (2.3) in the present paper 
and

$$
\begin{aligned}
\left\|C_{3}\right\| & \leqq \frac{1}{3 !}(\|A\|+\|B\|)^{3}\left[\frac{\partial^{3}}{\partial x^{3}} \exp \left(a_{1} x+a_{2} x^{2}\right)\right]_{x=0} ; \\
a_{1} & \equiv 2 .
\end{aligned}
$$

Thus, in general we obtain

$$
\left\|C_{n}\right\| \leqq a_{n} \cdot(\|A\|+\|B\|)^{n},
$$

where $a_{n}$ is defined recursively by

$$
a_{n}=\frac{1}{n !}\left[\frac{\partial^{n}}{\partial x^{n}} \exp \left(a_{1} x+a_{2} x^{2}+\ldots+a_{n-1} x^{n-1}\right)\right]_{x=0}
$$

for $n \geqq 2$.

One of the key points in our proof is to notice the following relation

$$
\exp \left(a_{1} x+a_{2} x^{2}+\ldots+a_{n} x^{n}\right)=1+a_{1} x+2\left(a_{2} x^{2}+\ldots+a_{n} x^{n}\right)+O\left(x^{n+1}\right) .
$$

This is easily proven from (2.12) by mathematical induction. Now, we introduce a function $f_{n}(x)$ defined by

$$
f_{n}(x)=a_{1} x+a_{2} x^{2}+\ldots+a_{n} x^{n} .
$$

Then $f_{n}(x)$ satisfies the relations

$$
\begin{aligned}
& \exp f_{n}(x)=1-a_{1} x+2 f_{n}(x)+\delta_{n}(x), \\
& \quad \delta_{n}(x)=O\left(x^{n+1}\right) \geqq 0 \text { and } \delta_{n}(0)=0 .
\end{aligned}
$$

Clearly, $f_{n}(0)=0$ and $f_{n}(x)$ is an increasing functional series for $x \geqq 0$, because all $a_{n}>0$. Furthermore, it is easily seen from Figure 1 that $f_{n}(x)$ is bounded from above as

$$
f_{n}(x) \leqq f(x)
$$

in the region $0 \leqq x \leqq \ln 2-\frac{1}{2}$, where $f(x)$ can be defined by a lower branch $(f(0)=0)$ of the solution

$$
\exp f(x)=1-a_{1} x+2 f(x)
$$

Thus, we arrive at the result that

$$
\lim _{n \rightarrow \infty} f_{n}(x) \equiv f_{\infty}(x)
$$

exists. On the other hand, from the definition of $a_{n}, f_{\infty}(x)$ satisfies the equation

$$
\exp f_{\infty}(x)=1-a_{1} x+2 f_{\infty}(x) ; \quad f_{\infty}(0)=0 .
$$

Therefore, we obtain that $f_{\infty}(x) \equiv f(x)$. That is,

$$
\lim _{n \rightarrow \infty} \delta_{n}(x)=0 \text { for } 0 \leqq x \leqq \ln 2-\frac{1}{2} \text {. }
$$




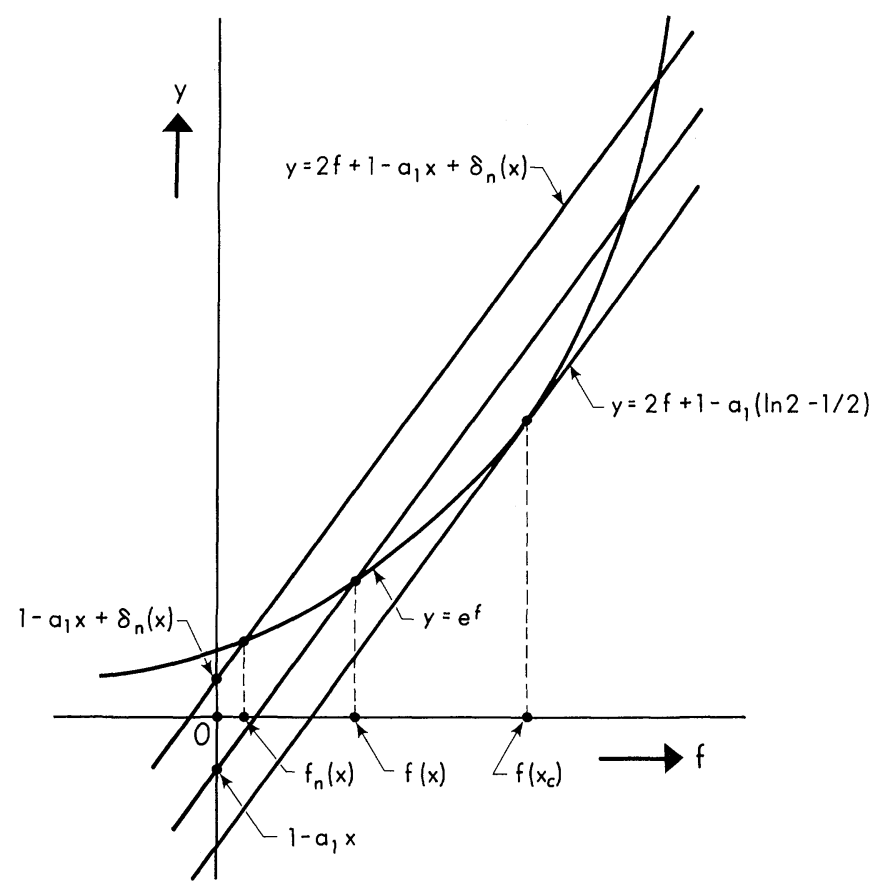

Fig. 1. A figure illustrating how to determine the functions $f_{n}(x)$ and $f(x)$ and to show the dependence of $f_{n}(x)$ upon $\delta_{n}(x)$

This relation yields the result that

$$
I(\lambda) \leqq|\lambda|(\|A\|+\|B\|)+\sum_{n=2}^{\infty} \lambda^{n} a_{n}(\|A\|+\|B\|)^{n}
$$

and that the right hand side of (2.21) converges for $|\lambda|(\|A\|+\|B\|) \leqq \ln 2-\frac{1}{2}$. We have also $f(x) \leqq \ln 2$ in the above region of $x$.

Next, we discuss the convergence of (2.1) with the use of the above result on $I(\lambda)$ and $\delta_{n}(x)$. Putting

$$
\Delta_{n}(\lambda) \equiv\left\|e^{\lambda(A+B)}-e^{\lambda A} e^{\lambda B} \ldots e^{\lambda^{n} C_{n}}\right\|,
$$

we have

$$
\begin{aligned}
\Delta_{n}(\lambda) & \leqq\left\|e^{\lambda A} e^{\lambda B} \ldots e^{\lambda^{n} C_{n}}\right\| \cdot\left\|e^{\lambda(A+B)} e^{-\lambda^{n} C_{n}} \ldots e^{-\lambda B} e^{-\lambda A}-1\right\| \\
& \leqq[\exp I(|\lambda|)] P(\lambda) .
\end{aligned}
$$

Here

$$
P(\lambda)=\left\|\mathscr{P}_{n}\left(e^{\lambda(A+B)} e^{-\lambda^{n} C_{n}} \ldots e^{-\lambda B} e^{-\lambda A}\right)\right\|
$$

with the use of a projection operator $\mathscr{P}_{n}$ introduced in $[I]$, which is defined $[I]$ by

$$
\mathscr{P}_{n}(f(\lambda)) \equiv f(\lambda)-\sum_{k=0}^{n} \frac{\lambda^{k}}{k !} f^{(k)}(0) .
$$


Now, we have

$$
\begin{aligned}
P(\lambda) & \leqq \mathscr{P}_{n} \exp \left[2|\lambda|(\|A\|+\|B\|)+|\lambda|^{2}\left\|C_{2}\right\|+\ldots+|\lambda|^{n}\left\|C_{n}\right\|\right] \\
& \leqq \mathscr{P}_{n} \exp \left[\sum_{k=1}^{n} a_{k}|\lambda|^{k}(\|A\|+\|B\|)^{k}\right] \\
& =\mathscr{P}_{n}\left[1-a_{1} \Lambda+2 f_{n}(\Lambda)+\delta_{n}(\Lambda)\right]=\delta_{n}(\Lambda),
\end{aligned}
$$

for $\Lambda \leqq \ln 2-\frac{1}{2}$ with $\Lambda=|\lambda|(\|A\|+\|B\|)$, where we have used the relations (2.11) and (2.15). Thus, we arrive finally at the inequality (2.5). Since $\delta_{n}(\Lambda)$ goes to zero for $\Lambda \leqq \ln 2-\frac{1}{2}$ as $n \rightarrow \infty$, we obtain (2.6) under the condition that $|\lambda|(\|A\|+\|B\|)$ $\leqq \ln 2-\frac{1}{2}$. This is only a sufficient condition for the convergence of (2.6). It is, however, worthwhile to have proven explicitly the convergence of the Zassenhaus formula for the first time.

Quite similarly, Theorem 1 is easily extended to the following set of operators $A_{1}, A_{2}, \ldots A_{p}$ :

Theorem 2. For any set of operators $\left\{A_{j}\right\}$ in a Banach algebra,

$$
\lim _{n \rightarrow \infty} e^{\lambda A_{1}} e^{\lambda A_{2}} \ldots e^{\lambda A_{p}} e^{\lambda^{2} C_{2}} \ldots e^{\lambda^{n} C_{n}}=\exp \left[\lambda \sum_{j=1}^{p} A_{j}\right]
$$

for $|\lambda| \sum_{j=1}^{p}\left\|A_{j}\right\| \leqq \ln 2-\frac{1}{2}$, where $C_{n}$ are now defined by

$$
C_{2}=\frac{1}{2}\left[\frac{\partial^{2}}{\partial \lambda^{2}}\left(e^{-\lambda A_{p}} \ldots e^{-\lambda A_{1}} e^{\lambda\left(A_{1}+\ldots+A_{p}\right)}\right)\right]_{\lambda=0}
$$

and in general $C_{n}$ is determined recursively by

$$
C_{n}=\frac{1}{n !}\left[\frac{\partial^{n}}{\partial \lambda^{n}}\left(e^{-\lambda^{n-1} C_{n-1}} \ldots e^{-\lambda^{2} C_{2}} e^{-\lambda A_{p}} \ldots e^{-\lambda A_{1}} e^{\lambda\left(A_{1}+\ldots+A_{p}\right)}\right)\right]_{\lambda=0} .
$$

The proof of this theorem is quite analogous to that of Theorem 1.

\section{Systematic Approximants Formula of Exponential Operators}

As discussed in Section 1, the $n-m$ approximant $f_{n, m}(A, B)$ defined by $(1.1)$ with the coefficients $\left\{C_{n}\right\}$ in (2.2)-(2.4), is very useful in quantum physics. In connection with this, we have the following theorem.

Theorem 3. For any set of operators $A$ and $B$ in a Banach algebra,

$$
\left\|e^{A+B}-f_{n, m}(A, B)\right\| \leqq n \cdot 2^{n-1} \delta_{m}\left(n^{-1}[\|A\|+\|B\|]\right) \exp \left[n^{-1}(2-n)(\|A\|+\|B\|)\right],
$$

for $n^{-1}(\|A\|+\|B\|) \leqq \ln 2-\frac{1}{2}$. Under this condition,

$\lim _{m \rightarrow \infty} f_{n, m}(A, B)=e^{A+B}$ 
Proof. We put

$$
P \equiv\left\|e^{A+B}-f_{n, m}(A, B)\right\|
$$

as in $[I]$. Then, we have

$$
P \leqq n\|g\|[\max (\|g\|,\|h\|)]^{n-1} F_{m}\left(\frac{1}{n}\right),
$$

as was shown in $[I]$, where

$$
g=\exp \left[\frac{1}{2}(A+B)\right] \text { and } h=\left[f_{n, m}(A, B)\right]^{1 / n},
$$

and

$$
F_{m}(\lambda)=\mathscr{P}_{m}\left(\exp \left[2 \lambda(\|A\|+\|B\|)+\lambda^{2}\left\|C_{2}\right\|+\ldots+\lambda^{m}\left\|C_{m}\right\|\right]\right) .
$$

Using the result obtained in Section 2, we get

$$
F_{m}(\lambda) \leqq \delta_{m}[|\lambda|(\|A\|+\|B\|)]
$$

for $|\lambda|(\|A\|+\|B\|) \leqq \ln 2-\frac{1}{2}$. On the other hand, we have

$$
\begin{aligned}
& \|g\|[\max (\|g\|,\|h\|)]^{n-1} \leqq \exp (\|A\|+\|B\|) \exp \left[(n-1) \sum_{k=2}^{m} n^{-k}\left\|C_{k}\right\|\right] \\
& \exp \left[n^{-1}(2-n)(\|A\|+\|B\|)\right] \exp \left\{(n-1) f_{m}\left[\frac{1}{n}(\|A\|+\|B\|)\right]\right\} \\
& \leqq 2^{n-1} \exp \left[n^{-1}(2-n)(\|A\|+\|B\|),\right.
\end{aligned}
$$

for $|\lambda|(\|A\|+\|B\|) \leqq \ln 2-\frac{1}{2}$. Here we have used the upper bound of $f_{m}(x): f_{m}(x)$ $\leqq \ln 2$, which has been proven in Section 2 . Thus, we arrive finally at Theorem 3 , noting that $\lim _{m \rightarrow \infty} \delta_{m}(x)=0$.

Similarly we have the following theorem for more than two operators.

Theorem 4. For any set of operators $\left\{A_{j}\right\}$ in a Banach algebra,

$$
\begin{aligned}
& \left\|\exp \left(\sum_{j=1}^{p} A_{j}\right)-f_{n, m}\left(\left\{A_{j}\right\}\right)\right\| \\
& \leqq n \cdot 2^{n-1} \delta_{m}\left(n^{-1} \sum_{j=1}^{p}\left\|A_{j}\right\|\right) \exp \left(n^{-1}(2-n) \sum_{j=1}^{p}\left\|A_{j}\right\|\right)
\end{aligned}
$$

where $f_{n, m}\left(\left\{A_{j}\right\}\right)$ is defined by

$$
f_{n, m}\left(\left\{A_{j}\right\}\right)=\left(e^{A_{1} / n} e^{A_{2} / n} \ldots e^{A_{p} / n} e^{C_{2} / n^{2}} \ldots e^{n^{-m} C_{m}}\right)^{n},
$$


and $\left\{C_{k}\right\}$ are given by (2.28) and (2.29).

$$
\begin{aligned}
& \text { For } n^{-1} \sum_{j=1}^{p}\left\|A_{j}\right\| \leqq \ln 2-\frac{1}{2}, \\
& \lim _{m \rightarrow \infty} f_{n, m}\left(\left\{A_{j}\right\}\right)=\exp \left(\sum_{j=1}^{p} A_{j}\right) .
\end{aligned}
$$

The proof of this theorem is quite the same as that of Theorem 3 .

\section{The Baker-Campbell-Hausdorff Formula and Related Exponential Operators}

The $\mathrm{BCH}$ formula takes the following form

$$
e^{\lambda A} e^{\lambda B}=e^{Z(\lambda)} \quad \text { and } \quad Z(\lambda)=\sum_{n=1}^{\infty} \lambda^{n} Z_{n}
$$

where $[12,13]$

$$
Z_{1}=A+B, Z_{2}=\frac{1}{2}[A, B], Z_{3}=\frac{1}{6}\left[Z_{2}, B-A\right], Z_{4}=\frac{1}{12}\left[\left[Z_{2}, A\right], B\right],
$$

and in general

$$
Z_{n}=\frac{1}{n !}\left[\frac{\partial^{n}}{\partial \lambda^{n}} \ln \sum_{k=0}^{\infty} \sum_{j=0}^{\infty} \frac{\lambda^{k+j}}{k ! j !} A^{k} B^{j}\right]_{\lambda=0} .
$$

We have the following theorem:

Theorem 5. The BCH formula converges, namely (4.1) converges for $|\lambda|(\|A\|+\|B\|)$ $<\ln 2$.

Proof. Clearly the following expansion

$$
e^{\lambda A} e^{\lambda B}=1+\lambda(A+B)+\ldots \equiv 1+F(\lambda)
$$

converges for any set of bounded operators $A$ and $B$ in a Banach algebra. Then, $Z(\lambda)$ is given by

$$
Z(\lambda)=\ln [1+F(\lambda)]=\sum_{n=1}^{\infty} \lambda^{n} Z_{n}
$$

This is convergent when $\|F(\lambda)\|<1$. This condition is satisfied if $|\lambda|(\|A\|+\|B\|)$ $<\ln 2$.

Similarly it is also useful to introduce the following expansion

$$
e^{\lambda(A+B)}=e^{\lambda A} e^{\lambda B} e^{W(\lambda)} ; \quad W(\lambda)=\sum_{n=2}^{\infty} \lambda^{n} W_{n},
$$

where $W_{2}=C_{2}, W_{3}=C_{3}, W_{4}=C_{4}$ with $\left\{C_{n}\right\}$ defined in (2.2), (2.3) and (2.4), and in general

$$
W_{n}=\frac{1}{n !}\left[\frac{\partial^{n}}{\partial \lambda^{n}} \ln \sum_{k=0}^{\infty} \sum_{j=0}^{\infty} \sum_{m=0}^{\infty} \frac{\lambda^{k+j+m}(-B)^{k}(-A)^{j}(A+B)^{m}}{k ! j ! m !}\right]_{\lambda=0} .
$$


We obtain easily the following theorem:

Theorem 6. For any set of operators $A$ and $B$ in a Banach algebra, the expansion (4.5) converges, at least, for

$$
|\lambda|(\|A\|+\|B\|)<\frac{1}{2} \ln 2 .
$$

The proof of this theorem is given in the same way as for Theorem 5 .

Acknowledgements. The author would like to thank Professor H. Araki for suggestive comments on [1], which have given the author a motive to investigate this problem, and also he would like to thank Professor D. D. Betts for a kind reading of the manuscript and also thank Professor D. D. Betts and Professor Y. Takahashi for their hospitality at Edmonton.

\section{References}

1. Suzuki, M.: Commun. math. Phys. 51, 183 (1976)

2. Magnus, W.: Commun. Pure Appl. Math. 7, 649 (1954)

3. Wilcox, R.M. : J. Math. Phys. 8, 962 (1967)

4. Trotter,H.F.: Proc. Am. Math. Soc. 10, 545 (1959); see also references cited in [I]

5. Suzuki, M. : Progr. Theoret. Phys. 56, 1454 (1976)

6. Suzuki, M., Miyashita,S., Kuroda, A., Kawabata,C.: Phys. Lett. 60 A, 478 (1977)

7. Suzuki, M., Miyashita,S., Kuroda,A.: Progr. Theoret. Phys. (submitted)

8. Rogiers, J., Dekeyser, R. : Phys. Rev. B 13, 4886 (1976)

9. Rogiers,J., Betts,D.D.: Physica 85 A, 553 (1976)

10. Brower, R.C., Kuttner,F., Nauenberg, M., Subbarao,K. : Preprint

11. Honda, N.: Private communication

12. Weiss, G.H., Maradudin, A. A. : J. Math. Phys. 3, 771 (1962)

13. Richtmyer, R.D., Greenspan, S.: Commun. Pure Appl. Math. 18, 107 (1965)

Communicated by $\mathrm{H}$. Araki

Received August 1, 1977 